\title{
INVENTORY OF WELLS NEAR A FORMER \\ WASTE-DISPOSAL SITE, NASHUA, \\ NEW HAMPSHIRE, JUNE 1993
}

By SARAH M. FLANAGAN

U.S. GEOLOGICAL SURVEY

Open-File Report 94-398

Prepared in cooperation with the

U.S. ENVIRONMENTAL PROTECTION AGENCY

REGION I

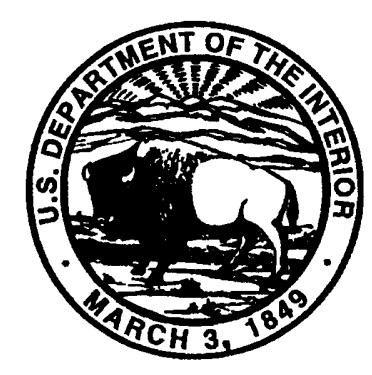

Bow, New Hampshire

1994 


\title{
U.S. DEPARTMENT OF THE INTERIOR \\ BRUCE BABBIT, Secretary
}

\author{
U.S. GEOLOGICAL SURVEY
}

Gordon P. Eaton, Director

\section{For additional information write to:}

District Chief

New Hampshire-Vermont District

U.S. Geological Survey

Water Resources Division

525 Clinton Street

Bow, New Hampshire 03304
Copies of this report can be purchased from:

U.S. Geological Survey

Earth Science Information Center Open-File Reports Section

Box 25286, MS 517

Federal Center

Denver, CO 80225-0425 


\section{CONTENTS}

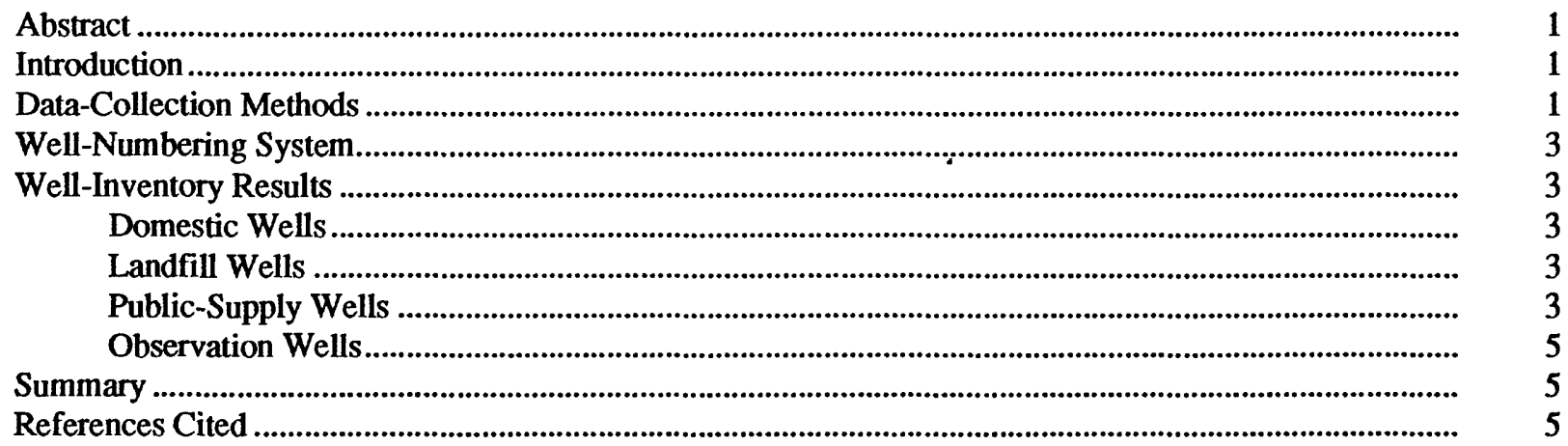

\section{ILLUSTRATIONS}

1. Map showing location of the well-inventory study area, the Gilson Road waste-disposal site, and the Nashua "Four Hills" Landfill in Hollis and Nashua, New Hampshire.

2. Map showing location of domestic, public-supply, and observation wells in and near the study area in Hollis and Nashua, New Hampshire.

\section{TABLES}

1. Records of selected wells and one boring in Hollis and Nashua, New Hampshire

2. Lithologic logs of selected wells and one boring in Hollis and Nashua, New Hampshire. 


\section{CONVERSION FACTORS AND VERTICAL DATUM}

\begin{tabular}{rcl}
\hline Multiply & By & To obtain \\
\hline foot $(\mathrm{ft})$ & 0.3048 & meter \\
gallon per minute $(\mathrm{gal} / \mathrm{min})$ & 0.06308 & liter per second \\
inch (in.) & 25.4 & millimeter \\
mile (mi) & 1.609 & kilometer \\
square mile $\left(\mathrm{mi}^{2}\right)$ & 2.59 & square kilometer \\
\hline
\end{tabular}

\section{Vertical Datum}

Sea Level: In this report, "sea level" refers to the National Geodetic Vertical Datum of 1929--a geodetic datum derived from a general adjustment of the first-order level nets of the United States and Canada, formerly called Sea Level Datum of 1929. 


\title{
Inventory Of Wells Near A Former Waste-Disposal Site, Nashua, New Hampshire, June 1993
}

\author{
By Sarah M. Flanagan
}

\begin{abstract}
Domestic, public-supply, and observation wells near a former waste-disposal site along Gilson Road in Nashua, New Hampshire, were inventoried in June 1993. Data include hydrogeologic information on 13 domestic wells, 11 observation wells in an adjacent municipal landfill, 3 abandoned public-supply wells, 3 observation wells, and 1 test boring drilled by the U.S. Geological Survey.
\end{abstract}

\section{INTRODUCTION}

Domestic, public-supply, and observation wells near a former waste-disposal site along Gilson Road in Nashua, New Hampshire, were inventoried in June 1993. This inventory was done by the U.S. Geological Survey (USGS) in cooperation with the U.S. Environmental Protection Agency (USEPA), Region I.

During the 1960's and early 1970's, disposal of hazardous and nonhazardous liquid waste around a privately owned garage along Gilson Road caused local ground-water contamination (Weston, 1989). In 1981, chemical analyses of ground-water samples from domestic wells downgradient from the Gilson Road site indicated that the wells were contaminated with volatile organic compounds (VOC's) and other hazardous substances (Weston, 1989). The site is currently undergoing remediation under the authority of the Comprehensive Environmental Response, Compensation, and Liability Act (CERCLA) of 1980.

The purpose of this report is to (1) inventory the location and status of existing domestic, land-fill, public-supply, and observation wells in the study area, and (2) list available hydrogeologic data associated with these wells. The area of the inventory was limited to about $1.7 \mathrm{mi}^{2}$ near the Gilson Road waste-disposal site in a residential area in southwestern Nashua, New Hampshire (fig. 1). During the 1980's, the population expanded rapidly because of the increase in trailer parks and single-family homes. In addition, the municipal landfill north of, and adjacent to, the Gilson Road waste-disposal site is currently (1993) undergoing expansion (fig. 1).

The major surface-water bodies in the study area include Lyle Reed and Trout Brooks, tributaries to the Nashua River. The Nashua River is a major tributary to the Merrimack River. The town of Hollis is just west of the study area across the Nashua River (fig. 1). The State and town boundaries between Nashua, New Hampshire, and Pepperell, Massachusetts, are less than $1 \mathrm{mi}$ to the south of the study area.

The surficial geology of the Pepperell Quadrangle in which the study area is located, was mapped at a scale of $1: 24,000$ by Koteff and Volkmann (1973). The hydrogeology of the Nashua area was studied by Toppin (1987) as part of a regional study to map the stratified-drift aquifers and determine their basic hydraulic properties.

The authors thank the personnel from the Nashua Public Works Department for assisting in the location of observation wells in the "Four Hills" landfill, and a private drilling company for providing aquifer-test data.

\section{DATA-COLLECTION METHODS}

Private homes were surveyed door-to-door in the study area, and resident responses from 13 homes are compiled in table 1 (at back of report). Observation wells in the Nashua "Four Hills" Landfill, which were 


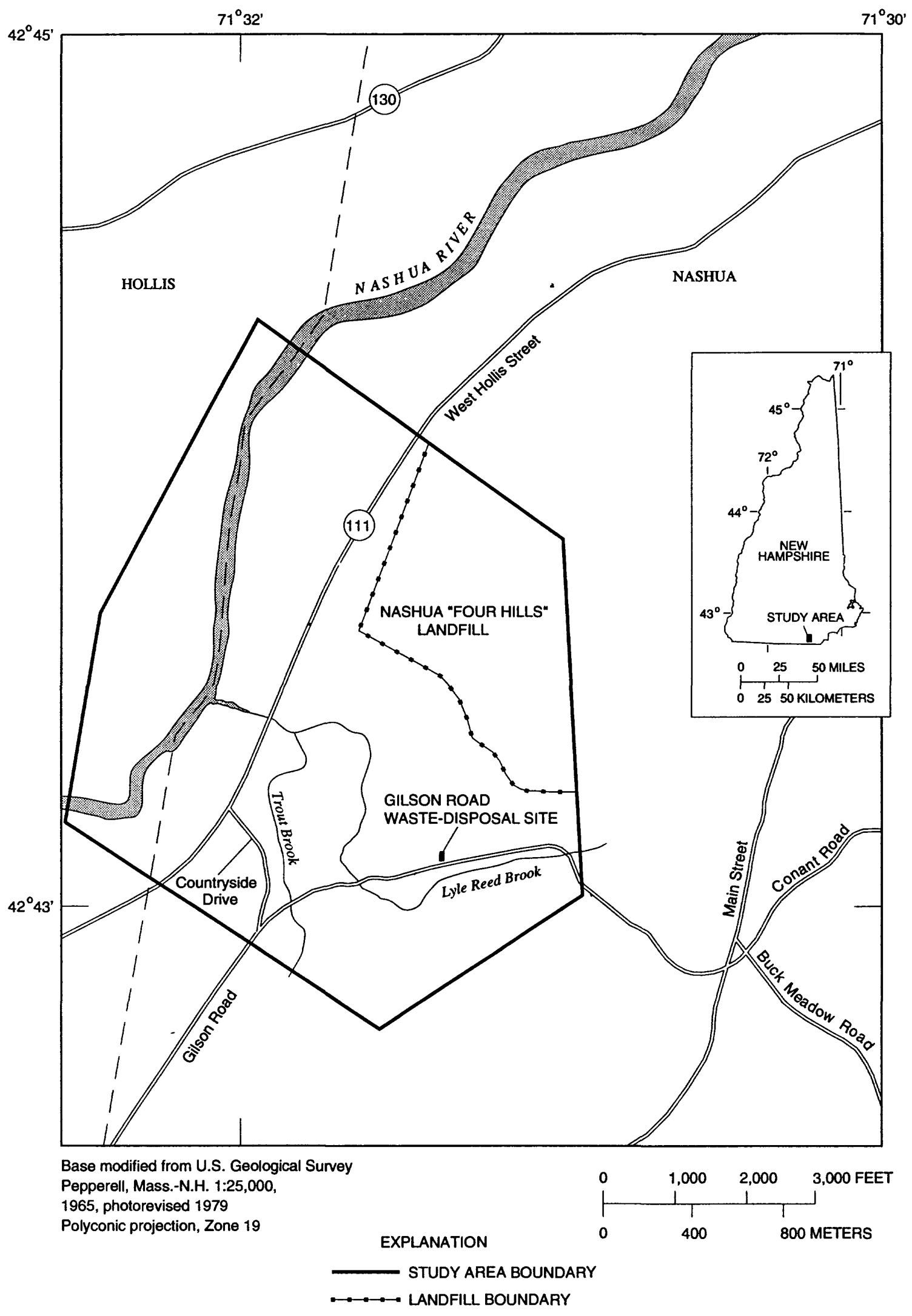

Figure 1. Location of the well-inventory study area, the Gilson Road waste-disposal site, and the Nashua "Four Hills" Landfill, in Hollis and Nashua, New Hampshire. 
within the boundary of the study area (fig. 1), also were inventoried and field checked during the survey. In addition, original well-log, construction, and aquifertest data were obtained for an abandoned public-supply well once owned by a private water company in the study area.

\section{WELL-NUMBERING SYSTEM}

Local numbers assigned to wells and test borings consist of a two-letter town designation- "HS" for Hollis and "NA" for Nashua; a supplemental letter designation (" $\mathrm{A}$ ", borings for hydrogeologic purposes with no casing set and " $W$ ", for all wells in which a casing was set); and a sequential number in each town. For example, the 220th well inventoried in Nashua is designated NAW-220.

\section{WELL-INVENTORY RESULTS}

The hydrogeologic data presented in this section include site records, well-construction records, exploration-borehole logs, and aquifer-test field data. A summary of the well inventory is presented in the following sections for domestic, landfill, public-supply, and observations wells (table 2, at back of report). Locations of inventoried wells and borings were plotted on the 1:25,000-scale Pepperell, Massachusetts-New Hampshire USGS topographic map and are shown in figure 2. Lithologic logs for 18 wells and 1 exploration borehole are summarized in table 2.

\section{Domestic Wells}

Of the 25 private homes surveyed, 9 are still connected to domestic wells, 3 switched to a private watersupply company but still have existing domestic wells in place, 12 switched to a private water-supply company and removed their domestic wells, and 1, across the Nashua River in Hollis (HSW-70), has a bedrock well that is used exclusively for restroom facilities (for a private hunting club).

Attempts to obtain hydrogeologic data for domestic wells from the 25 homeowners were mostly unsuccessful. Residents who still used their own wells knew the well depth but not the depth to bedrock or depth of casing, with the exception of the homeowner for well NAW-227.

\section{Landfill Wells}

About 35 observation wells were installed in the Nashua "Four Hills" Landfill from 1983 to 1992. Eleven of these wells were within the boundary of the study area and were inventoried as part of this study (fig. 2). The well in the landfill that is closest to and downgradient from the Gilson Road waste-disposal site is well NAW-237 (also known as MW-5).

\section{Public-Supply Wells}

During the 1960's, three public-supply wells were in use in the inventory area and downgradient from the Gilson Road waste-disposal facility (fig. 2). All three wells have since been removed from service, and their well casings were removed during the early 1970's. Two of the three wells (NAW-157 and NAW-158) were owned by a local trailer park and installed in 1959. Attempts to obtain hydrogeologic data for these two wells were unsuccessful.

A third, public-supply well (NAW-220) was owned and operated by a private water-supply company on land owned by a local trailer park. This 24-inchdiameter gravel-packed well was drilled in November 1969. A 192-hour aquifer test, using five observation wells, was done from December 1-9, 1969, and a 3-day recovery test was done after the pump was shut off. A constant volume of $1,210 \mathrm{gal} / \mathrm{min}$ of ground water was pumped from well NAW-220 for the duration of the aquifer test resulting in a total drawdown at the well of $29.29 \mathrm{ft}$ (unpublished data on file with the Bow, New Hampshire office of the U.S. Geological Survey). In 1976 or 1977 , the decision was made to abandon the well (Bernard Rousseau, Pennichuck Water Works, oral commun., 1993). Written documentation is not available about the conditions around the well at the time the decision was made to abandon the well and remove the casing. 


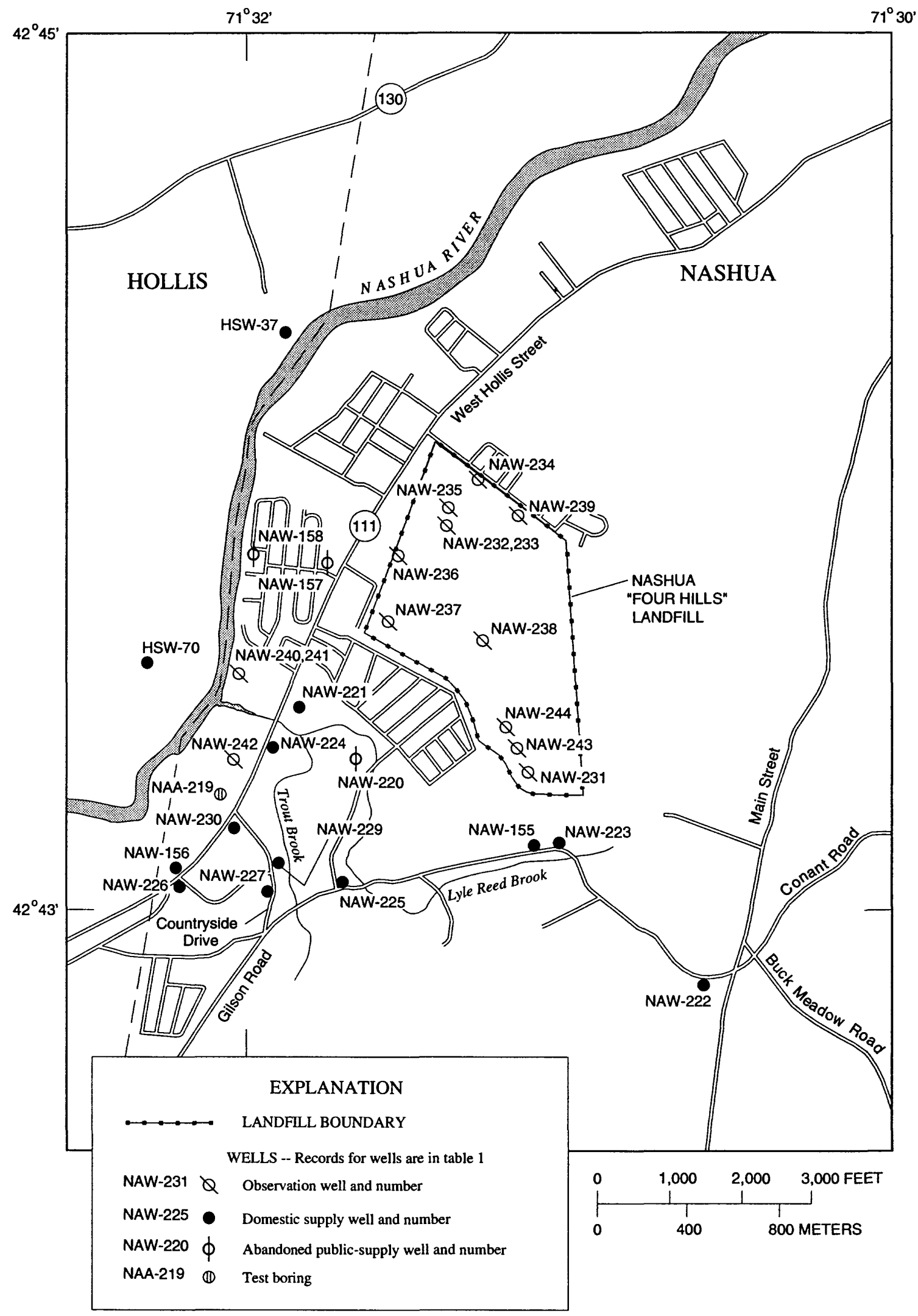

Figure 2. Location of domestic, public-supply, and observation wells in and near the study area in Hollis and Nashua, New Hampshire. 


\section{Observation Wells}

On July $12-13,1993$, test-well drilling was done by the U.S. Geological Survey on private property west of West Hollis Street near the present-day storage facilities (J.D. Ayotte, U.S. Geological Survey, written commun., 1993). Three test sites were selected for drilling-wells NAW-240 (29 ft) and NAW-241 (14 ft) nested together at one site, well NAW-242 at another site, and boring NAA-219 at the third site (fig. 2).

\section{SUMMARY}

An inventory of 13 domestic, 3 abandoned publicsupply, and 14 observation wells, and 1 boring was completed in June 1993 near a former waste-disposal site along Gilson Road in Nashua, New Hampshire. Hydrogeologic data, including aquifer-test data, were obtained for one of the former public-supply wells
(NAW-220). Lithologic, ground-water level, wellconstruction, and other data were collected and tabulated.

\section{REFERENCES CITED}

Koteff, Carl and Volkmann, R.P., 1973, Surficial geologic map of the Pepperell Quadrangle, Middlesex County, Massachusetts and Hillsborough County, New Hampshire: U.S. Geological Survey, Geologic Quadrangle Map GQ-1118, scale 1:24,000.

Toppin, K.W., 1987, Hydrogeology of stratified-drift aquifers and water quality in the Nashua Regional Planning Commission Area, South-Central New Hampshire: U.S. Geological Survey Water-Resources Investigations Report 86-4358, 45 p., 6 pl.

Weston, Roy F., Inc., 1989, Remedial program evaluation, Gilson Road site, Nashua, New Hampshire: Concord, N.H. 
Table 1. Records of selected wells and one boring in Hollis and Nashua, New Hampshire

[Site No.: First two characters are the town code (HS for Hollis, New Hampshire; NA for Nashua, New Hampshire). Third Latitude, Longitude: Accurate to 5 seconds. Type of well: Bor, test boring; BrW, bedrock well; Dug, dug well; Dvn, driven unused. Water level: reported as feet below land surface; gal/min, gallon per minute]

\begin{tabular}{|c|c|c|c|c|c|c|c|c|}
\hline Site No. & $\begin{array}{l}\text { Latitude } \\
\bullet ' \prime \prime\end{array}$ & Longitude & Owner's name & $\begin{array}{c}\text { Other } \\
\text { identification }\end{array}$ & $\begin{array}{c}\text { Year } \\
\text { completed }\end{array}$ & $\begin{array}{l}\text { Type } \\
\text { of well }\end{array}$ & $\begin{array}{l}\text { Use of } \\
\text { water }\end{array}$ & $\begin{array}{l}\text { Depth } \\
\text { of well }\end{array}$ \\
\hline \multicolumn{9}{|c|}{ Hollis } \\
\hline $\begin{array}{l}\text { HSW-37 } \\
\text { HSW-70 }\end{array}$ & $\begin{array}{l}424419 \\
424334\end{array}$ & $\begin{array}{l}713152 \\
713218\end{array}$ & $\begin{array}{l}\text { Gagnon } \\
\text { Lone Pine Hunting Club }\end{array}$ & $\begin{array}{l}-- \\
-\end{array}$ & 1955 & $\begin{array}{l}\text { Dug } \\
\text { BrW }\end{array}$ & $\begin{array}{l}\mathrm{H} \\
\mathrm{H}\end{array}$ & $\begin{array}{l}14.1 \\
--\end{array}$ \\
\hline \multicolumn{9}{|c|}{ Nashua } \\
\hline $\begin{array}{l}\text { NAA-219 } \\
\text { NAW-155 } \\
\text { NAW-156 } \\
\text { NAW-157 } \\
\text { NAW-158 }\end{array}$ & $\begin{array}{l}424315 \\
424307 \\
424306 \\
424347 \\
424349\end{array}$ & $\begin{array}{l}713205 \\
713107 \\
713213 \\
713144 \\
713158\end{array}$ & $\begin{array}{l}\text { Dobbens, John } \\
\text { Poirier, Camil } \\
\text { Lagios, } \\
\text { Jensen's Trailer Park } \\
\text { do. }\end{array}$ & $\begin{array}{l}-- \\
-- \\
-- \\
- \\
-\end{array}$ & $\begin{array}{r}1993 \\
-\overline{1} \\
1936 \\
1959 \\
1959\end{array}$ & $\begin{array}{l}\text { Bor } \\
\text { Dug } \\
\text { Dug } \\
\text { GPW } \\
\text { GPW }\end{array}$ & $\begin{array}{l}U \\
U \\
H \\
U \\
U\end{array}$ & $\begin{array}{l}-- \\
20.3 \\
53.1 \\
45 \\
80\end{array}$ \\
\hline NAW-220 & 424321 & 713139 & Pennichuck Water Works & Jensen W-1 & 1969 & GPW & $U$ & 67.5 \\
\hline $\begin{array}{l}\text { NAW-221 } \\
\text { NAW-222 }\end{array}$ & $\begin{array}{l}424327 \\
424250\end{array}$ & $\begin{array}{l}713151 \\
713035\end{array}$ & $\begin{array}{l}\text { Morey, Mary } \\
\text { Kane, Maryanne }\end{array}$ & $\overline{165.0023}$ & $\begin{array}{l}1943 \\
1987\end{array}$ & $\begin{array}{l}\mathrm{BrW} \\
\mathrm{BrW}\end{array}$ & $\begin{array}{l}\mathbf{H} \\
\mathbf{H}\end{array}$ & $\begin{array}{l}300 \\
200\end{array}$ \\
\hline NAW-223 & 424308 & 713102 & Taylor, Richard & -- & 1957 & Dug & $\mathrm{H}$ & 20 \\
\hline NAW-224 & 424325 & 713156 & Shaw, Steven & 6 & - & Dug & $\mathrm{U}$ & -- \\
\hline $\begin{array}{l}\text { NAW-225 } \\
\text { NAW-226 } \\
\text { NAW-227 } \\
\text { NAW-229 } \\
\text { NAW-230 }\end{array}$ & $\begin{array}{l}424308 \\
424303 \\
424305 \\
424307 \\
424314\end{array}$ & $\begin{array}{l}713142 \\
713213 \\
713156 \\
713155 \\
713202\end{array}$ & $\begin{array}{l}\text { Lambert, Romeo } \\
\text { Humphry, Lawrence \& Ellen } \\
\text { Benson, Alfred } \\
\text { Whynott, Bob (renter) } \\
\text { Fortier, William J. }\end{array}$ & $\begin{array}{l}7 \\
19 \\
\text { B-3S } \\
\text { MW-1 } \\
\text { MW-1S }\end{array}$ & $\begin{array}{r}1970 \\
1972 \\
1964 \\
1-\overline{1} \\
1963\end{array}$ & $\begin{array}{l}\text { Dvn } \\
\text { BrW } \\
\text { BrW } \\
\text { Dug } \\
\text { BrW }\end{array}$ & $\begin{array}{l}\mathrm{H} \\
\mathrm{H} \\
\mathrm{H} \\
\mathrm{H} \\
\mathrm{H}\end{array}$ & $\begin{array}{r}15 \\
150 \\
200 \\
150\end{array}$ \\
\hline NAW-231 & 424319 & 713106 & Nashua "Four Hills" Landfill & MW-3 & 1990 & Obs & $\mathrm{U}$ & 30 \\
\hline $\begin{array}{l}\text { NAW-232 } \\
\text { NAW-233 }\end{array}$ & $\begin{array}{l}424352 \\
424352\end{array}$ & $\begin{array}{l}713122 \\
713122\end{array}$ & $\begin{array}{l}\text { do. } \\
\text { do. }\end{array}$ & $\begin{array}{l}\text { MW-4 } \\
\text { MW-5 }\end{array}$ & $\begin{array}{l}1983 \\
1990\end{array}$ & $\begin{array}{l}\text { Obs } \\
\text { Obs }\end{array}$ & $\mathrm{U}$ & $\begin{array}{l}93.5 \\
49.5\end{array}$ \\
\hline $\begin{array}{l}\text { NAW-234 } \\
\text { NAW-235 }\end{array}$ & $\begin{array}{l}424358 \\
424355\end{array}$ & $\begin{array}{l}713116 \\
713122\end{array}$ & $\begin{array}{l}\text { do. } \\
\text { do. }\end{array}$ & MW-7 & $\begin{array}{l}1983 \\
1983\end{array}$ & $\begin{array}{l}\text { Obs } \\
\text { Obs }\end{array}$ & $\mathrm{U}$ & $\begin{array}{l}45 \\
85\end{array}$ \\
\hline $\begin{array}{l}\text { NAW-236 } \\
\text { NAW-237 } \\
\text { NAW-238 } \\
\text { NAW-239 } \\
\text { NAW-240 }\end{array}$ & $\begin{array}{l}424349 \\
424340 \\
424337 \\
424354 \\
424333\end{array}$ & $\begin{array}{l}713131 \\
713134 \\
713115 \\
713109 \\
713200\end{array}$ & \begin{tabular}{|l} 
do. \\
do. \\
do. \\
do. \\
Dobbens, Don
\end{tabular} & $\begin{array}{l}\text { MW-8 } \\
-- \\
-- \\
\ddot{B}-4\end{array}$ & $\begin{array}{l}1983 \\
1983 \\
1983 \\
1992 \\
1993\end{array}$ & $\begin{array}{l}\text { Obs } \\
\text { Obs } \\
\text { Obs } \\
\text { Obs } \\
\text { Obs }\end{array}$ & $\begin{array}{l}U \\
U \\
U \\
U \\
U\end{array}$ & $\begin{array}{l}33 \\
76 \\
14 \\
28 \\
29\end{array}$ \\
\hline $\begin{array}{l}\text { NAW-241 } \\
\text { NAW-242 } \\
\text { NAW-243 } \\
\text { NAW-244 }\end{array}$ & $\begin{array}{l}424333 \\
424322 \\
424322 \\
424326 \\
\end{array}$ & $\begin{array}{l}713200 \\
713203 \\
713110 \\
713112\end{array}$ & $\begin{array}{l}\text { do. } \\
\text { do. } \\
\text { Nashua "Four Hills" Landfill } \\
\text { do. }\end{array}$ & $\begin{array}{l}\text { B-15-T } \\
-- \\
-- \\
--\end{array}$ & $\begin{array}{l}1993 \\
1993 \\
1993 \\
1993\end{array}$ & $\begin{array}{l}\text { Obs } \\
\text { Obs } \\
\text { Obs } \\
\text { Obs }\end{array}$ & $\begin{array}{l}\mathbf{U} \\
\mathbf{U} \\
\mathbf{U} \\
\mathbf{U}\end{array}$ & $\begin{array}{c}14 \\
30 \\
84.5 \\
132 \\
\end{array}$ \\
\hline
\end{tabular}


character-W, indicates well; A, indicates boring. The numbers are sequential in the order the wells were inventoried.

well; GPW, gravel packed well; Obs; observation well. USGS: U.S. Geological Survey. Use of water: H, domestic use; U,

\begin{tabular}{|c|c|c|c|c|c|c|c|c|c|}
\hline \multirow[b]{2}{*}{ Site No. } & \multicolumn{3}{|c|}{ Casing } & \multicolumn{2}{|c|}{ Screened interval (ft) } & \multicolumn{3}{|c|}{ Water level } & \multirow[b]{2}{*}{ Remarks } \\
\hline & $\begin{array}{l}\text { Height } \\
\text { above } \\
\text { ground } \\
\text { (ft) }\end{array}$ & $\begin{array}{l}\text { Depth } \\
\text { to } \\
\text { bottom } \\
\text { (ft) }\end{array}$ & $\begin{array}{l}\text { Dia- } \\
\text { meter } \\
\text { (in.) }\end{array}$ & $\begin{array}{c}\text { Top of } \\
\text { opening }\end{array}$ & $\begin{array}{c}\text { Bottom } \\
\text { to } \\
\text { opening }\end{array}$ & $\begin{array}{l}\text { Altitude } \\
\text { above } \\
\text { sea level. }\end{array}$ & Date & $\begin{array}{l}\text { Depth } \\
\text { (ft) }\end{array}$ & \\
\hline
\end{tabular}

\begin{tabular}{|c|c|c|c|c|c|c|c|c|c|}
\hline \multicolumn{10}{|c|}{ Hollis } \\
\hline $\begin{array}{l}\text { HSW-37 } \\
\text { HSW-70 }\end{array}$ & -- & - & 12 & - & - & $\begin{array}{l}175 \\
185\end{array}$ & $6-20-63$ & 8.5 & $\ddot{\text { Nat uced for drinking water }}$ \\
\hline \multicolumn{10}{|c|}{ Nashua } \\
\hline $\begin{array}{l}\text { NAA-219 } \\
\text { NAW-155 } \\
\text { NAW-156 } \\
\text { NAW-157 } \\
\text { NAW-158 }\end{array}$ & $\begin{array}{l}- \\
- \\
- \\
- \\
-\end{array}$ & $\begin{array}{l}- \\
-- \\
-- \\
- \\
-\end{array}$ & $\begin{array}{r}- \\
36 \\
8 \\
36 \\
8\end{array}$ & $\begin{array}{l}-- \\
- \\
- \\
-\end{array}$ & $\begin{array}{l}- \\
- \\
- \\
- \\
-\end{array}$ & $\begin{array}{l}185 \\
193 \\
197 \\
195 \\
170\end{array}$ & $\begin{array}{r}7-13-93 \\
7-16-93 \\
7-31-62 \\
-- \\
--\end{array}$ & $\begin{array}{l}14 \\
15.8 \\
21.4 \\
-- \\
--\end{array}$ & $\begin{array}{l}\text { Boring; drilled by USGS } \\
\text { Not in use } \\
\text { Destroyed in } 1992 . \\
\text { Not in use, casing removed } \\
\text { Not in use, casing removed }\end{array}$ \\
\hline NAW-220 & 2.5 & 52 & 24 & 52 & 67.5 & 170 & $11-01-69$ & 8 & $\begin{array}{l}\text { Originally used for public supply. } \\
\text { During aquifer test, well was } \\
\text { pumped } 1,210 \mathrm{ga} / \mathrm{min} \text { for } 192 \\
\text { hours from Dec. } 1-9,1969 \text {. Casing } \\
\text { was removed in } 1976-77 \text { because of } \\
\text { ground-water contamination. } \\
\text { Drilled by R.E. Chapman }\end{array}$ \\
\hline $\begin{array}{l}\text { NAW-221 } \\
\text { NAW-222 }\end{array}$ & $\overline{1.0}$ & $\overline{60}$ & $\begin{array}{l}6 \\
6\end{array}$ & - & -- & $\begin{array}{l}180 \\
210\end{array}$ & $\begin{array}{r}10-08-87 \\
--\end{array}$ & 25 & $\begin{array}{l}\text { In use } \\
\text { Owner reports "hard water" from } \\
\text { well. Drilled by Skillings }\end{array}$ \\
\hline NAW-223 & -- & -- & -- & -- & -- & 190 & $6-08-93$ & 10.3 & $\begin{array}{l}\text { Dug well in cellar of house. Owner } \\
\text { also uses a driven point well about } \\
300 \mathrm{ft} \text { from house. Water from this } \\
\text { well is used for the garden and lawn } \\
\text { Not in use }\end{array}$ \\
\hline $\begin{array}{l}\text { NAW-225 } \\
\text { NAW-226 } \\
\text { NAW-227 } \\
\text { NAW-229 } \\
\text { NAW-230 }\end{array}$ & $\begin{array}{l}-- \\
-- \\
- \\
-\end{array}$ & $\begin{array}{c}-- \\
\overline{35} \\
--\end{array}$ & $\begin{array}{l}-- \\
- \\
6 \\
- \\
--\end{array}$ & $\begin{array}{l}- \\
- \\
- \\
- \\
-\end{array}$ & $\begin{array}{l}- \\
-- \\
-- \\
-- \\
-\end{array}$ & $\begin{array}{l}190 \\
210 \\
190 \\
180 \\
195\end{array}$ & $\begin{array}{l}- \\
- \\
- \\
-- \\
-\end{array}$ & $\begin{array}{l}-- \\
-- \\
-- \\
-- \\
--\end{array}$ & $\begin{array}{l}\text { In use } \\
\text { Do. } \\
\text { Do. } \\
\text { In use; in cellar of house } \\
\text { In use }\end{array}$ \\
\hline NAW-231 & 3.29 & 10 & 1.5 & 10 & 30 & 199.37 & $12-19-90$ & 12.5 & $\begin{array}{l}\text { A bedrock well also is nested here. } \\
\text { Drilled by All Terrain Drilling }\end{array}$ \\
\hline $\begin{array}{l}\text { NAW-232 } \\
\text { NAW-233 }\end{array}$ & $\begin{array}{r}.54 \\
3.29\end{array}$ & $\begin{array}{l}90 \\
39.5\end{array}$ & $\begin{array}{l}1.5 \\
1.5\end{array}$ & $\begin{array}{l}90 \\
39.5\end{array}$ & $\begin{array}{l}93.5 \\
49.5\end{array}$ & $\begin{array}{l}187.67 \\
188.53\end{array}$ & $\begin{array}{r}12-19-90 \\
2-11-92\end{array}$ & $\begin{array}{l}22.8 \\
20.9\end{array}$ & $\begin{array}{l}\text { Drilled by Soils Exploration } \\
\text { Shallow well nested with NAW- } \\
232 \text { (MW-1) Drilled by All Terrain } \\
\text { Drilling }\end{array}$ \\
\hline $\begin{array}{l}\text { NAW-234 } \\
\text { NAW-235 }\end{array}$ & $\begin{array}{r}.65 \\
1.24\end{array}$ & $\begin{array}{r}5 \\
15\end{array}$ & $\begin{array}{l}2 \\
2\end{array}$ & $\begin{array}{r}5 \\
15\end{array}$ & $\begin{array}{l}45 \\
85\end{array}$ & $\begin{array}{l}173.6 \\
185.3\end{array}$ & $\begin{array}{l}12-19-90 \\
12-19-83\end{array}$ & $\begin{array}{r}4.3 \\
18.1\end{array}$ & $\begin{array}{l}\text { Drilled by Soils Exploration } \\
\text { Do. }\end{array}$ \\
\hline $\begin{array}{l}\text { NAW-236 } \\
\text { NAW-237 } \\
\text { NAW-238 } \\
\text { NAW-239 } \\
\text { NAW-240 }\end{array}$ & $\begin{array}{l}2.41 \\
1.95 \\
4.22 \\
3.24 \\
--\end{array}$ & $\begin{array}{c}13 \\
6 \\
4.5 \\
20 \\
26.5\end{array}$ & $\begin{array}{l}2 \\
2 \\
2 \\
1.5 \\
2\end{array}$ & $\begin{array}{l}13 \\
6 \\
4.5 \\
20 \\
26.5\end{array}$ & $\begin{array}{l}33 \\
76 \\
14 \\
28 \\
29\end{array}$ & $\begin{array}{l}178.3 \\
177.9 \\
184.6 \\
177.14 \\
165\end{array}$ & $\begin{array}{r}12-19-90 \\
12-19-90 \\
1-09-91 \\
2-11-92 \\
7-13-93\end{array}$ & $\begin{array}{l}10.5 \\
10.1 \\
8.77 \\
.9 \\
11\end{array}$ & $\begin{array}{l}\text { Do. } \\
\text { Do. } \\
\text { Do. } \\
\text { Do. } \\
\text { Drilled by USGS }\end{array}$ \\
\hline $\begin{array}{l}\text { NAW-241 } \\
\text { NAW-242 } \\
\text { NAW-243 } \\
\text { NAW-244 }\end{array}$ & $\begin{array}{l}-- \\
- \\
\overline{3} .0\end{array}$ & $\begin{array}{r}11.5 \\
27.5 \\
1 \overline{117}\end{array}$ & $\begin{array}{l}2 \\
2 \\
1.5 \\
1.5\end{array}$ & $\begin{array}{l}11.5 \\
27.5 \\
11 \overline{7}\end{array}$ & $\begin{array}{c}14 \\
30 \\
84.5 \\
132\end{array}$ & $\begin{array}{l}165 \\
185 \\
235 \\
305\end{array}$ & $\begin{array}{r}7-13-93 \\
7-13-93 \\
-- \\
-\end{array}$ & $\begin{array}{c}11 \\
21 \\
-- \\
--\end{array}$ & $\begin{array}{l}\text { Do. } \\
\text { Do. } \\
\text { Drilled by Soils Exploration } \\
\text { Do. }\end{array}$ \\
\hline
\end{tabular}


Table 2. Lithologic logs of selected wells and one boring in Nashua, New Hampshire

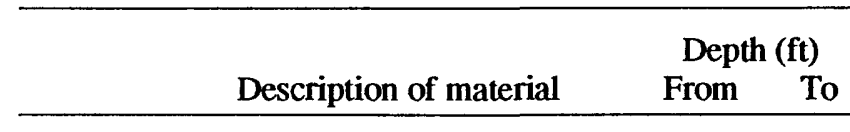

\section{NAA-219}

Sand, fine to very fine; few pebble

$0-7$

Silt and sand till; few pebbles and cored cobbles $7-16$

Refusal, firm; probably bedrock 16

\section{NAW-155}

Sand and gravel

Bedrock

$$
0-20
$$$$
20
$$

\section{NAW-220}

Sand, fine, brown $0-30$

Sand, fine to medium

Sand, coarse and medium gravel.

Gravel, coarse

Bottom of hole.

NAW-222

Sand and gravel

Bedrock

$0-9$

$50-200$

NAW-223

Sand and gravel

$0-20$

Bottom of hole

20

NAW-224

Sand and gravel

0

NAW-225

Sand $0-15$

Bottom of hole 15

\section{NAW-231}

Sand, brown, organic

Sand, fine to medium, brown; trace of silt

Sand, fine to coarse, brown

Silt, brown, and fine sand

Sand, fine, brown

Silt, brown, and fine sand

Till, sandy, gravelly; some silt and cobbles...... $32.3-36.6$

Bedrock (cored 6 in.)

$0-0.5$

$0.5-3$

$3-15.7$

$15.7-24$

$24-28$

$28-32.3$

36.6

\section{NAW-232}

Sand, fine to medium, dark brown; some silt....

Sand, fine to medium, light brown

Sand, fine, brown

Till (possible, no recovery)

Bedrock (cored $5 \mathrm{ft}$ )

\begin{tabular}{|c|c|}
\hline & Depth (ft) \\
\hline Description of material & From To \\
\hline
\end{tabular}

NAW-234

Top soil, reddish-brown, fine sand and pebbles $\quad \begin{array}{lll}0 & - & 2\end{array}$

Sand, fine, light brown; little silt ........................ $2 \quad 2 \quad 8$

Silt, light brown; little fine sand ...................... $8 \quad 8 \quad 24$

Sand, fine to medium, light brown; little silt; trace of gravel................................................ $24 \quad 30$

Sand, fine to medium, and silt, light brown...... $30 \quad-33.5$

Sand, medium to coarse, light brown;

few gravel

$33.5-36$

Sand, fine, light brown; few gravel..................... $36 \quad-40$

Sand, fine to medium, light brown .................... $40 \quad-50$

Till, silt and gray fine sand, some pebbles;

trace of clay

$50-56$

Bottom of hole 56

\section{NAW-235}

Fill, fine sand and silt, dark brown; pebbles..... $0 \quad 0 \quad-2$

Sand, fine to coarse, brown; trace of silt............ 2 - 10

Sand, fine, light brown....................................... 10 - 45

Sand, fine to medium, light brown ................... $45 \quad-75$

Sand, fine, light brown..................................... $75 \quad-83$

Till; silt, brown-gray; fine sand; little clay; pebbles.

$83-85$

Bottom of hole

\section{NAW-236}

Top soil, silt and fine sand, dark brown............. 0

Sand, fine to medium, light brown ................... 1 - 25

Sand, fine to coarse, light brown; few gravel... $25 \quad-33$

Till, silt, gray fine sand, pebbles;

very compact

$33-37.5$

Bottom of hole 37.5

NAW-237

Sand, medium to coarse, light brown

$0-8.5$

Sand and gravel, medium to coarse, light brown.

$8.5-14$

Sand and gravel, medium to coarse, light brown; some rock fragments................. $14 \quad-19$

Sand and gravel, coarse, brown ........................ $19 \quad-24$

Sand, coarse, brown ............................................ $24 \quad-29$

Sand, medium to coarse, brown........................ $29 \quad 49$

Sand, medium to coarse, brown; trace of silt.... $49 \quad-60$

Sand and gravel, medium to coarse, brown; trace of silt.

Sand, fine to medium, brown; trace of silt......... $67 \quad-74$

Sand, fine to coarse, brown; little silt ................ $74 \quad-75.5$

Till, silt to fine sand and pebbles, gray-brown

Bottom of hole 
Table 2. Lithologic logs of selected wells and one boring in Nashua, New Hampshire--Continued

\begin{tabular}{cc} 
Description of material & \multicolumn{2}{c}{ Depth (ft) } \\
From & To \\
\hline
\end{tabular}

NAW-238

Till, silt, sand, and pebbles

Bedrock (possible); cored $6.5 \mathrm{ft}$

$0-7.5$

7.5

\section{NAW-239}

Sand, brown, and organic silt

Sand, fine to medium, brown; some coarse sand; little silt

Gravel, brown, and fine to coarse sand; some cobbles; little silt.

Sand, fine to medium, brown; trace of silt and coarse sand

Gravel, and fine to medium sand, brown; little silt and coarse sand.

Bedrock (cored $5 \mathrm{ft}$ )

$0-0.7$

$0.7-2$

$2-8$

$8-18.4$

$18.4-29.3$

$29.3-35$

NAW-240

Sand, very fine to fine, $\tan$; coarser with depth

$0-10$

Sand, medium to coarse, tan; moderately to well sorted

$10-21$

$21-31$

Sand, fine with some coarser layers..

Till; loosely compacted, mostly coarse sand.

Refusal, firm; probably bedrock

\begin{tabular}{ccc}
\hline & \multicolumn{2}{c}{ Depth (ft) } \\
Description of material & From & To \\
\hline
\end{tabular}

NAW-242

Sand and silt, very fine..................................... $0 \quad-12$

Till, sandy ........................................................ 12 - 15

Sand, very fine and silt .................................... $15 \quad-24$

Till; loosely compacted; more compact with depth.

$24-33.5$

NAW-243

Tree stump....................................................... 0 - 1.4

Sand, gravel, clay, silt ......................................... 1.49

Till, sandy, gravelly, clayey, silty ..................... 9 - 84.5

Bottom of hole ......................................................... 84.5

\section{NAW-244}

Till; fine-medium silty sand; gravel and

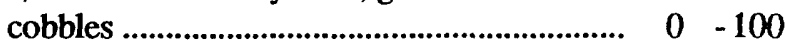

Till; same as above, but more cobbles ................. $100 \quad-125$

Boulder ............................................................. 125 - 126

Till, many cobbles; weathered bedrock............. $126 \quad-137$

Bedrock (cored $3 \mathrm{ft}$ ) ........................................... $137 \quad-140$ 